\section{Genetic algorithm aided timeslot scheduling for UTRA TDD CDMA networks}

\section{S. Ni and L. Hanzo}

It is demonstrated that genetic algorithms may be utilised for finding a suboptimum but highly beneficial uplink (UL) or downlink (DL) timeslot (TS) allocation for improving the achievable performance of the third generation UTRA system's time division duplex (TDD) mode. It is demonstrated that this novel GA-assisted UL/DL timeslot scheduling scheme is capable of avoiding the severe BS to BS intercell interference potentially inflicted by the UTRA TDD CDMA air interface owing to allowing all TSs to be used both in the UL and DL, hence potentially quadrupling the carried teletraffic and halving the uplink power.

Introduction: Although the UTRA time division duplex (TDD) mode was contrived for the sake of improving the achievable network performance by assigning all the timeslots on a demand basis to the uplink (UL) and downlink (DL), this mode may result in an excessive BS-to-BS interference and hence in a potentially reduced number of system users. More explicitly, it was shown that the TDD mode is more prone to avalanche-like teletraffic overload and its carried teletraffic is up to a factor two lower than that of the FDD mode [1]. To mitigate this deficiency, in this Letter genetic algorithms (GAs) [2] are proposed for improving the achievable performance of the UTRA TDD mode, potentially quadrupling the teletraffic carried, and simultaneously halving the average uplink power consumption required.

System model: The aim of this design is to maximise the achievable UTRA-like TDD/CDMA network's normalised carried traffic expressed in units of erlang $/ \mathrm{km}^{2} / \mathrm{MHz}$. The performance metrics used to quantify the quality of service have been described in [1]. The optimisation task to be solved by the GA is to find the best UL/DL constellation of all the timeslots (TSs) of all the cells for all UTRA TDD frames. Naturally, this optimisation task would impose an excessive complexity and hence a computationally efficient suboptimum solution is sought.

A simple example of the possible timeslot allocation scenarios is given in Fig. 1, portraying four different timeslot allocation scenarios for two BSs and two MSs. More specifically, in the scenario of Figs. 1a and $c, B S_{1}$ experiences two types of inter-cell interference, namely $M S_{2} \rightarrow B S_{1}$ and $B S_{2} \rightarrow B S_{1}$, respectively. Similarly, in the scenario seen in Figs. $1 b$ and $d, M S_{1}$ also experiences two different types of inter-cell interferences imposed by the neighbouring cell, which manifests itself as $M S_{2} \rightarrow M S_{1}$ and $B S_{2} \rightarrow M S_{1}$ interference, respectively. In [3] it was shown that the major source of interference is constituted by the BS-to-BS interference, as a consequence of the BS's high signal power and the near-LOS propagation conditions prevailing between the high-elevation BSs. Hence we can avoid encountering a high $B S \rightarrow B S$ inter-cell interference by appropriately scheduling the allocation of timeslots. Interference is inherent in cellular systems, and it is a challenging task to control it in practice due to the presence of random propagation effects. If there are $n$ potential serving BSs in an area, for each timeslot there are $2 n$ ways of allocating it to a specific BS either in the uplink or downlink. As argued before, an optimal timeslot allocation algorithm would have to tentatively invoke all $2^{n}$ possible TS allocations, to find the best one, when a new TS has to be allocated to a user, who is initiating a new call. However, since this new TS allocation affects the entire system's interference patterns, the complexity of the optimum full-search algorithm would become excessive. To reduce the complexity of the associated decisions, we invoked a genetic algorithm for determining the advantageous scheduling of uplink and downlink timeslots. The genetic algorithm uses an objective function [2] to determine how 'fit' each UL/DL TS allocation is for 'survival' in the consecutive generations of the GA, when ranking the potential UL/DL TS allocations. For instance, the aim of the GA is to determine in the example provided in Fig. 1 which particular UL/DL TS allocation of the total of four different options has the best overall connection quality, lower UL/DL average power consumption and lower interference level. The GA's objective function constituted by the number of users supported will be evaluated for a small fraction of the entire set of possible TS allocations, while aiming for a near-optimum solution. All simulation parameters conformed to those of the UTRA TDD network [2]. The GA's parameters are summarised in Table 1.

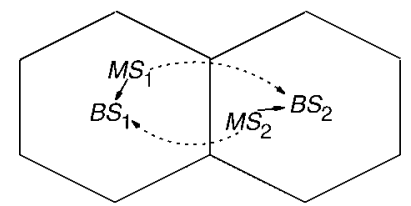

a

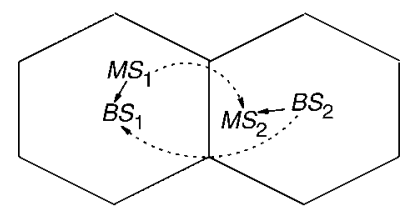

$c$

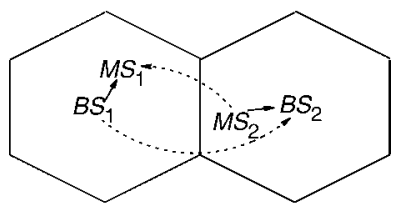

$b$

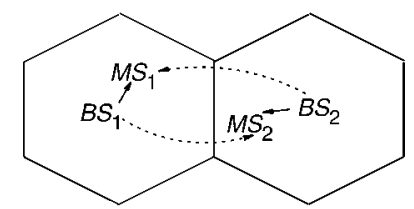

$d$ timeslot ID: 1

number of BSs: 2 number of MSs: 2

$\longrightarrow$ desired signals inter-cell interference

Fig. 1 Example of $U L / D L$ timeslot allocation options

Table 1: Configuration of GA used

\begin{tabular}{|c|c|}
\hline Set-up/Parameter & Method/Value \\
\hline Individual initialisation method & Uniform random \\
\hline Selection method & Fitness-proportionate \\
\hline Crossover operation & Single point \\
\hline Mutation operation & Uniform random bit flip \\
\hline Population size & $P=10$ \\
\hline Generation size & $Y=10$ \\
\hline Probability of mutation & 0.1 \\
\hline Probability of crossover & 0.9 \\
\hline Computational complexity & $P Y=100$ \\
\hline
\end{tabular}

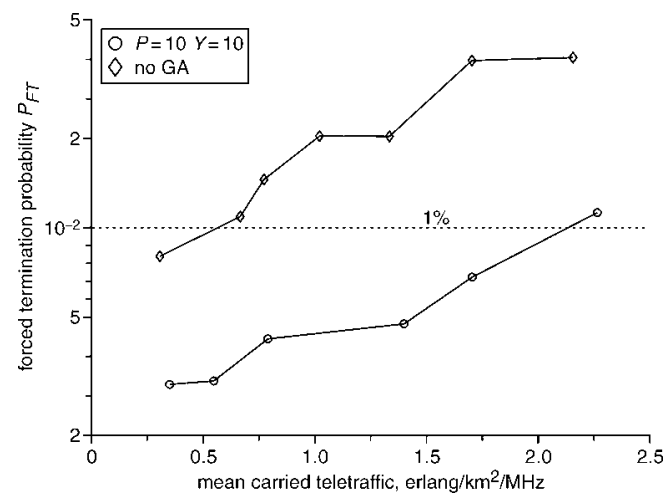

Fig. 2 Call dropping probability against mean carried traffic of UTRA-like TDD/CDMA based cellular network both with and without GA-assisted timeslots allocation as well as with shadowing having a standard deviation of $3 \mathrm{~dB}$ for $S F=16$

Simulation results: Fig. 2 shows the forced termination probability associated with a variety of traffic loads quantified in terms of the mean normalised carried traffic expressed in erlang $/ \mathrm{km}^{2} / \mathrm{MHz}$, when subjected to $0.5 \mathrm{~Hz}$ frequency shadowing having a standard deviation of $3 \mathrm{~dB}$. As observed in Fig. 2, nearly an order of magnitude forced termination probability $\left(P_{F T}\right)$ reduction has been achieved by employing GA-assisted timeslots scheduling compared to the 'no GA' scheme refraining from using UL/DL TS optimisation. Alternatively, at $P_{F T}=1 \%$ the traffic carried is approximately quadrupled. This is because the fixed timeslot allocation using no GA-aided TS-scheduling may inflict a high $B S \rightarrow B S$ interference, when the serving cell is using uplink timeslots and the interfering cell is using downlink timeslots, as shown in Figs. $1 b$ or $c$. The associated high inter-cell interference may result in a poor SINR, which fails to satisfy the system's target SINR required for maintaining a high-quality connection and hence increases the probability of forced termination. By 
contrast, in the GA-assisted UTRA TDD/CDMA system each timeslot in a frame can be allocated to either the uplink or downlink, depending on the associated slot-SINR, potentially allowing us to allocate the timeslot by minimising the inter-cell interference inflicted. As mentioned in the preceeding Section, for a UTRA/ TDD system having $n$ BSs, there are $2^{n}$ possible UL/DL TS allocation schemes for each timeslot. In our simulated scenario there are 49 wrapped-around traffic cells, as in [4], creating a search space of size $2^{49}$, although naturally a large fraction of the legitimate TS allocations are rather deficient. As argued before, the size of this search-space is excessive, preventing a full search. As a more attractive design option, a GA is utilised for finding a suboptimum but highly beneficial uplink or downlink TS allocation by searching only a fraction of these allocations. The computational complexity of the GA-aided search was set to $P Y=100$ objective function evaluations, where $P$ is population size and $Y$ is the number of generations. The 'no GA' based TDD network was found to support 58 users at $P_{F T}=1 \%$, corresponding to a traffic load of 0.6 erlang $/ \mathrm{km}^{2} / \mathrm{MHz}$. Upon employing GA-assisted UL/DL timeslot allocation before the transmission of each 15-slots UTRA TDD frame, the number of users supported by the TDD network increased to 185 users, or to an equivalent traffic load of $2.11 \mathrm{erlang} / \mathrm{km}^{2} / \mathrm{MHz}$, when invoking a GA population size of $P=10$ and $Y=10$ generations.

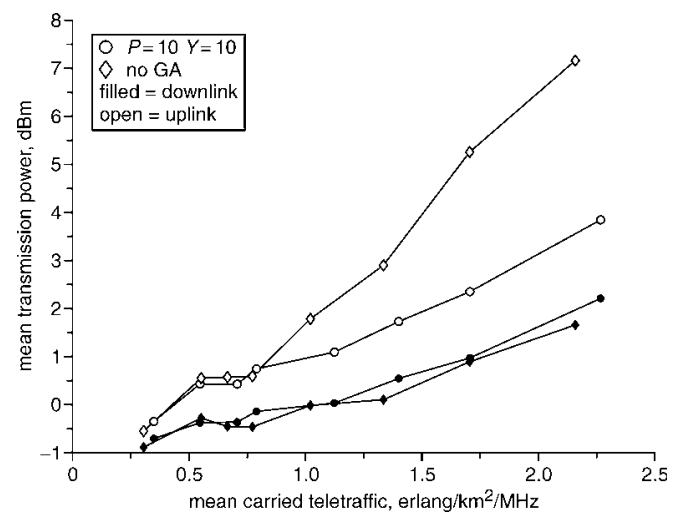

Fig. 3 Mean transmission power against mean carried traffic of UTRA-like TDD/CDMA based cellular network both with and without GA-assisted UL/DL TS-allocation as well as with shadowing having a standard deviation of $3 \mathrm{~dB}$ for a spreading factor of $S F=16$

For the sake of characterising the achievable system performance also from a different perspective, the mean transmission power against teletraffic performance is shown in Fig. 3. We observe in the Figure that both the 'GA-assisted' and 'no GA' scenario obey a similar trend in terms of their downlink power consumption. However, in terms of uplink power consumption, the 'no GA' scheme requires an average of $2-5 \mathrm{dBm}$ more signal power than the 'GA-assisted' scheme, as the traffic load becomes higher. Again, this is because the severe $B S \rightarrow B S$ inter-cell interference degrades the quality of the call. Hence, for the sake of achieving the target SINR and maintain the existing connections, the MSs have to increase their transmission power, which results in an increased interference level imposed on other connections, hence inflicting a performance degradation upon the whole system. The 'GAassisted' system is capable of avoiding the presence of severe interference by advantageously scheduling the UL/DL timeslots, hence keeping the system's average power as low as possible for the sake of supporting more MSs.

Conclusion: The UTRA TDD/CDMA system invoking GA-aided TS-scheduling approximately quadruples the carried traffic at $P_{F T}=1 \%$, while halving the required UL transmit power.

(C) IEE 2005

Electronics Letters online no: 20058185 doi: 10.1049/el:20058185

S. Ni and L. Hanzo (School of Electronics and Computer Science, University of Southampton, Southampton SO17 1BJ, United Kingdom)

E-mail: 1h@ecs.soton.ac.uk

\section{References}

1 Ni, S., Blogh, J.S., and Hanzo, L.: 'On the network performance of UTRA-like TDD and FDD CDMA systems using adaptive modulation and adaptive beamforming'. Proc. IEEE Vehicular Technology Conf. 2003, Spring, Jeju, Korea, April 2003, pp. 606-610

2 Hanzo, L., Yang, L.L., Kuan, E.L., and Yen, K.: 'Single- and multi-carrier DS-CDMA' (John Wiley and IEEE Press, New York, 2003)

3 Wu, X., Yang, L.-L., and Hanzo, L.: 'Uplink capacity investigations of TDD/CDMA'. Proc. IEEE Vehicular Technology Conf., VTC, Spring 2002, Birmingham, AL, USA, May 2002, Vol. 2, pp. 997-1001

4 Blogh, J.S., and Hanzo, L.: 'Third-generation systems and intelligent wireless networking-smart antennas and adaptive modulation' (John Wiley and IEEE Press, New York, 2002) 